\title{
Graphene Enhanced Wireless Sensors
}

\author{
Taoran Le, Trang Thai, Vasileios Lakafosis, \\ Manos Tentzeris \\ School of Electrical and Computer Engineering \\ Georgia Institute of Technology \\ Atlanta, GA, U.S.A. \\ taoran.le@ece.gatech.edu
}

\begin{abstract}
In this paper we demonstrate the design and development of a family of low-cost, self-powered, wireless sensor solutions utilizing both analog and digital principles. The sensors will utilize Graphene-based thin films integrated directly into the structure. For an immediately deployable digital sensing solution compatible with current commercial technologies we will utilize the Intel WISP platform, which can be read with current COTS products. Our thin films are produced from water-based, inkjet printed graphene oxide (GO) on paper/Kapton, developed using both conventional thermal and laser reduction techniques. In addition to reporting the first ever integration of inkjet-printed water soluble GO inks into low cost, flexible RF electronics, we also bring gas sensing capabilities to RFID tags relying on purely wireless digital transmission. The introduction of low cost, mass producible, eco-friendly, reduced graphene oxide (RGO) films on paper substrates lays the foundation for the development of a wide range of new low-cost, high performance Graphene-based electronic devices.
\end{abstract}

\section{INTRODUCTION}

The fast and reliable detection of poisonous gases is essential for personal and environmental safety. Therefore, much research has been devoted to this topic. Most recently, attention has been devoted to development of sensors deploying novel nano-materials, such as carbon nanotube and graphene, as the sensing medium. These materials alter their properties in the presence of a given substance due to their ability to absorb certain compounds. Chemical absorption produces changes in properties such as real and imaginary impedance, DC resistance, conductance, and effective dielectric constant. These changes can be exploited and monitored to determine the presence of various chemical compounds by translating the material effects into measurable electrical quantities. Most importantly, these materials make extremely sensitive sensors [1] capable of sensing substances down to the ppt level. However, much of the research focuses on development of wired platforms, such as resonators, transistors, and other basic circuit elements. Our focus is to develop truly wireless, passive sensing solutions capable of being deployed in harsh environments.

\author{
Ziyin Lin, Yunnan Fang, Kenneth Sandhage, \\ Chingping Wong \\ School of Materials Science and Engineering \\ Georgia Institute of Technology \\ Atlanta, GA, U.S.A. \\ zylin@gatech.edu
}

\section{INKJET PRINTING OF GRAPHENE -BASED GAS SENSOR PROTOTYPE}

The core of the wireless gas sensor is a prototype board made from graphene deposited onto Kapton substrate. Conductive silver ink is used to create the traces connecting the graphene pad to the external circuitry.

The first step in the sensor development process was the creation of stable, long-life, inkjet-printable graphene-based inks. This was accomplished by first converting the graphene into GO powder. Unlike pristine graphene which has very poor dispersion in common solvents, GO exhibits excellent solubility in water due to the existence of hydrophilic functional groups on the surface [2], rendering it an excellent candidate for development of eco-friendly water-based inks. After deposition, pure graphene was obtained by the reduction of GO, which reverts the conjugated basal plane and restores the electrical properties. The reduction of GO is considered as one of most promising methods for low-cost, high yield and scalable preparation of graphene materials [3].

\section{A. Creation of Graphene Oxide}

The GO was produced by chemical oxidation of graphite, which introduces oxygen-containing functional groups to exfoliate pristine graphite into individual GO sheets. GO was prepared with Hummers' method [4]. Graphite flake was placed into a $\mathrm{NaNO}_{3}$ /concentrated $\mathrm{H}_{2} \mathrm{SO}_{4}$ solution in an ice bath. Subsequently, $\mathrm{KMnO} 4$ was slowly added to the solution while maintaining the temperature below $20{ }^{\circ} \mathrm{C}$. The mixture was stirred in the ice bath for 2 hours and for another 30 minutes in $35{ }^{\circ} \mathrm{C}$ water bath. Next, $70{ }^{\circ} \mathrm{C}$ water was added drop-wise into the flask. The heat generated via exothermic reaction raised the solution temperature up to $98{ }^{\circ} \mathrm{C}$. Next, additional $70{ }^{\circ} \mathrm{C}$ water was then added, followed by hydrogen peroxide solution to terminate the reaction. The mixture was filtrated and washed with water to remove excess acid and inorganic salts. The resulting GO was dried overnight at $55^{\circ} \mathrm{C}$ to produce the GO powder. To prepare the GO ink, dry GO powder was dispersed in a water/glycerol solution and sonicated to disperse any aggregated particles. 


\section{B. Fabrication via Inkjet Printing on Kapton}

A Dimatix Materials Printer (DMP-2800) Series material deposition system was used to print the silver and the GO ink. The fabrication process involved deposition of 10 layers of conductive silver onto the Kapton substrate, followed by 42 layers of graphene oxide. First, the 10 layers of Cabot ${ }^{\circledR}$ silver ink were deposited and cured at $120^{\circ} \mathrm{C}$ for 8 hours. Next, 2 layers of GO were deposited and cured at $80^{\circ} \mathrm{C}$ for 4 hours to serve as a foundation for further prints. Next, the GO was deposited in 5 layer increments, with curing in between to ensure the highest consistency between samples. Deposition of the GO ink proved challenging at first, due to the hydrophobicity of the Kapton substrate. Therefore, before printing the Kapton substrate was treated by UVOzone to improve the hydrophilicity. The treated Kapton demonstrated better performance during printing, enabling higher resolution prints and more layers to be printed during a given cycle. Figure 1 below illustrates the printing of GO ink. Figure 2 shows the completed sensor elements prior to reduction of the GO pads.

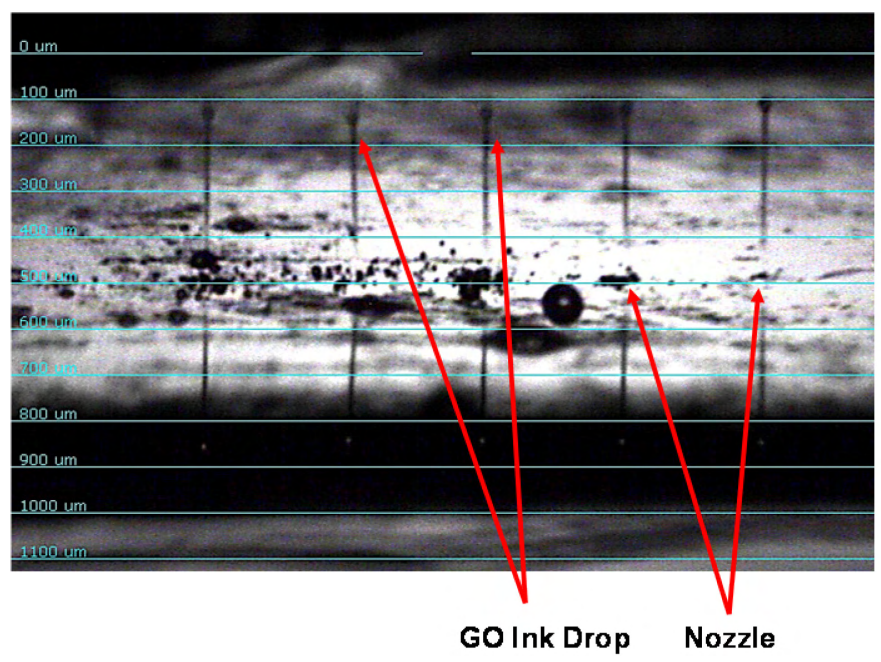

Figure 1. Graphene drop ejected from inkjet printer.

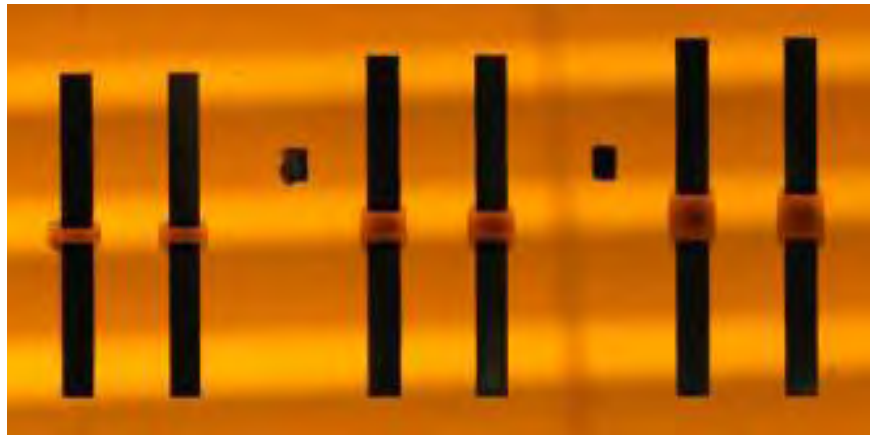

Figure 2. Graphene oxide thin films inkjet-printed in-between overlapping silver conductive inkjet-printed trace on Katpon substrate.

\section{Thermal Reduction of Graphene Oxide}

After printing and curing, each sample was reduced to obtain the desired graphene. Reduction was achieved by placing the printed GO in a hydrogen and argon atmosphere at elevated temperature. After reduction, the samples were left to cool down to room temperature naturally. The reduced graphene oxide layers were characterized via scanning electron microscopy (SEM) to determine their overall quality (i.e. presence of defects in the print, etc.). Figure 3 below provides an SEM image of the RGO thin film. As seen in Figure 3, wrinkles on the RGO surface are characteristics of $\mathrm{GO} / \mathrm{RGO}$.

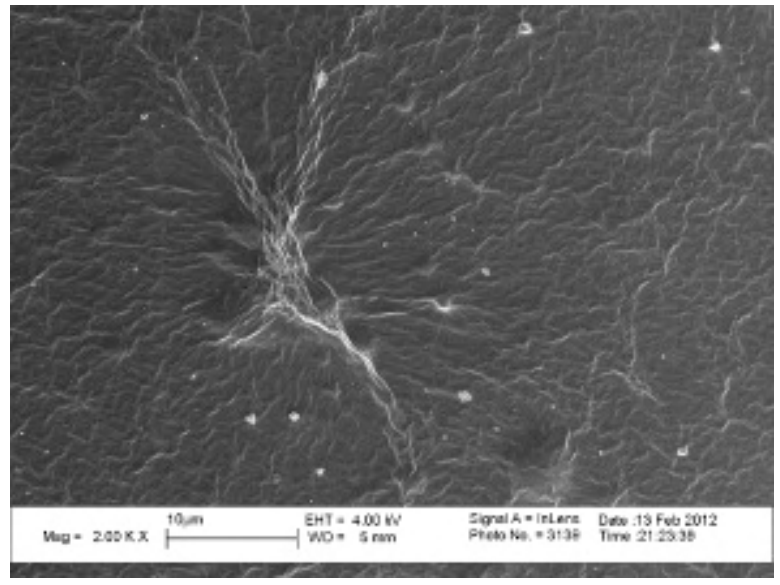

Figure 3. SEM image of the inkjet printed RGO thin film.

\section{BATTERY-LESS WIRELESS SENSING AND COMMUNICATION PLATFORM}

The WISP is a fully passive, battery-free and programmable RFID Tag [5] which can be powered and read by off-the-shelf EPC Gen2 UHF RFID Readers and has an on-board microcontroller for sensing and computing functions. In this work, we modify (hardware- and softwarewise) the WISP to create a sensing and communication platform that enables for the first time the introduction of gas sensing capabilities to RFID tags.

As opposed to the distortion-prone analog-based measurement techniques (with the most representative example being the resonant frequency shift sensing), this flexible platform offers the reliability of wireless digital transmission. Our developed prototype WISP-GS (Gas Sensor) platform is shown in Figure 4. The WISP-GS is solely powered by the RF energy illuminated by a very popular commercial RFID Reader. This $915 \mathrm{MHz}$ RF energy is rectified with its charge pump to charge the on-board capacitor. As soon as sufficient energy is accumulated and the voltage across the capacitor monitored by a supervisor exceeds the turn-on voltage of the MCU by an overhead margin, a regulated voltage of $1.805 \mathrm{~V}$ is used to power both the MCU and our prototype external gas sensing board. 
The external WISP-GS board consists, of course, of the graphene-based inkjet-printed RGO sensor, presented in the previous section, as well as the analog interface of this sensor.

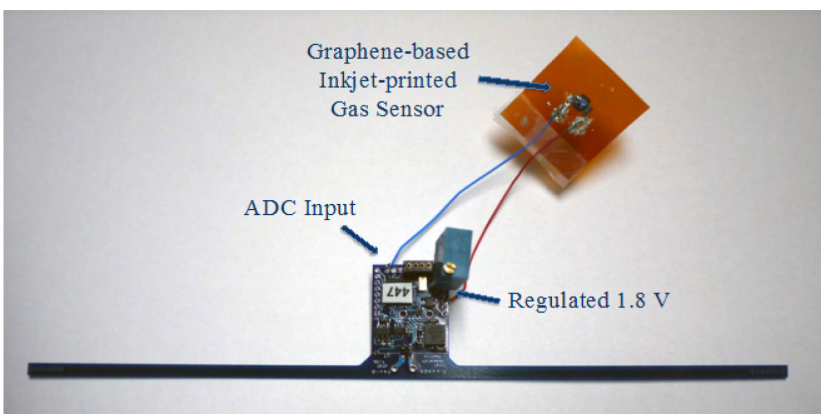

Figure 4. The WISP-GS (Gas Sensor) prototype platform

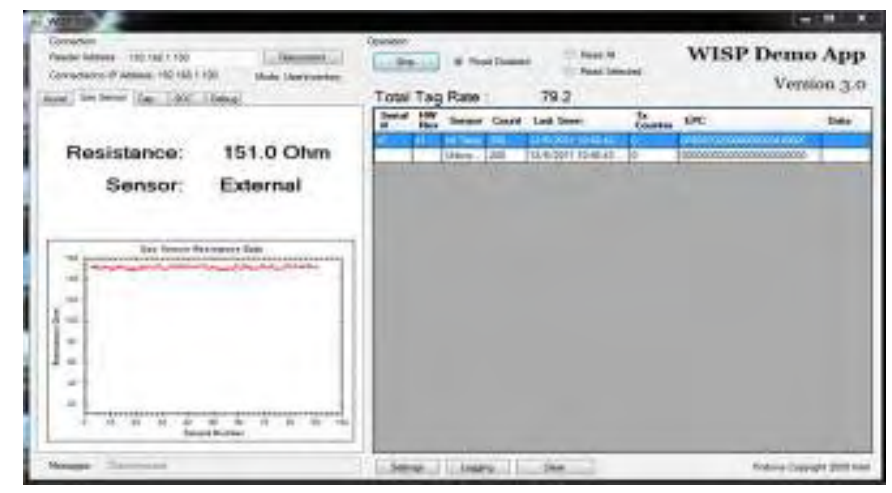

Figure 5. The WISP graphical user interface that captures and plots the sensed information from the transmitted RFID EPC message by the WISPGS Tag

For this very first prototype that serves as a proof of concept and because of the small range of the resistance change $(6 \%)$ relative to the initial resistance, the analog interface is essentially a voltage divider, in which the voltage drop across the gas sensor module is directly tied to the resistance change of the sensor's grapheme film pad and can easily be captured with the WISP's integrated Analog-toDigital Converter (ADC) (pin 2.3). In particular, the graphene-based micro-strip line's impedance is in parallel with one of the two resistors in series. Of course, special care had to be taken for the selection of the resistors of the voltage divider to not only keep the sensor board's peak consumption less than $2 \mathrm{~mW}$ but to also maximize, at the same time, the resolution given the particular impedance range that starts from the initial impedance of the graphene-based sensor samples in air and extends to the converged final impedance that corresponds to the application-dependent maximum concentration of the harmful gases under test. Additionally, the typically necessary zero offset has to be set with one-time simple software code adjustment prior to measurements with each gas sensor. The resolution achieved can be defined by dividing the maximum resistance value by the 1024 discrete levels of the integrated 10-bit ADC, which gives approximately $\pm 1 \mathrm{mV}$ accuracy.
Whenever located within the interrogation zone of an RFID reader, the WISP-GS is automatically detected and begins transmission of the sensed information within the EPC message of the RFID communication, as shown in Figure 5.

This figure shows a screenshot of our modified WISP graphical user interface that reports in real time the resistance of the gas film that can be easily mapped to the real gas concentration with the extracted fitting equation, the sensor's serial number and type, the total number of readings, the time it was last seen as well the full EPC Gen2 message transmitted. The report rate is only a few milliseconds, allowing for immediate reaction to health-threatening gas concentrations.

\section{EXPERIMENTAL SETUP}

The sensors were tested using an Environics ${ }^{\circledR}$ S4000 gas mixing system. The setup was capable of producing reliable mixtures up to $500 \mathrm{ppm}$ of ammonia gas diluted in air delivered at a rate of $50 \mathrm{ccm}$. To calibrate the sensor, the graphene samples were placed into a semi-closed glass chamber, which is shown in Figure 6, to direct the flow of gas onto the sensor. The custom designed gas flowing chamber has internal dimensions of $4 \mathrm{~cm} \times 1.5 \mathrm{~cm} \times 1.5 \mathrm{~cm}$. Air flowed through the system for 15 minutes to establish a system baseline. Next, $500 \mathrm{ppm}$ of ammonia/air mixture was introduced into the system and measurements of the resistance were taken at one minute internals for 15 minutes until near steady state condition in the material was achieved. Finally, the gas source was removed from the device while measurements continued to be taken for another 15 minute interval in order to measure the recovery time.

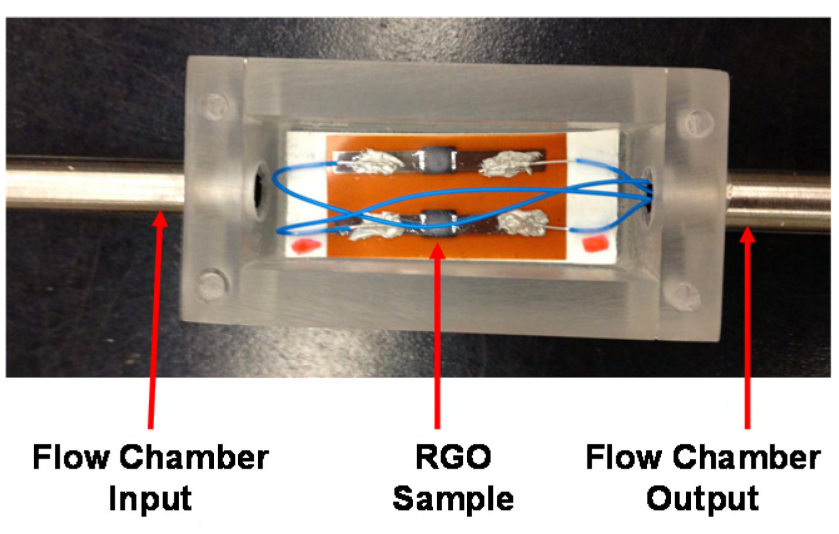

Figure 6. Measurement setup of the graphene sample.

\section{EXPERIMENTAL RESULTS}

The results of gas sensing experiments are shown in Figure 7. The inkjet-printed RGO with different dimensions show similar responses to $500 \mathrm{ppm} \mathrm{NH}_{3}$. The electrical resistance increases quickly after the introduction of $\mathrm{NH}_{3}$, indicating a fast detection rate. With the continued supply of $\mathrm{NH}_{3}$, the resistance change begins to diminish until reaching 
a saturation region after $\sim 10$ mins. A maximum sensitivity is observed in the case of Pattern 3, which shows greater than $6 \%$ increase in normalized resistance. After the introduction of $\mathrm{NH}_{3}$, pure air was introduced into the system and it was found that the resistance quickly recovered a large portion of its original value $(\sim 30 \%)$ within 5 mins.

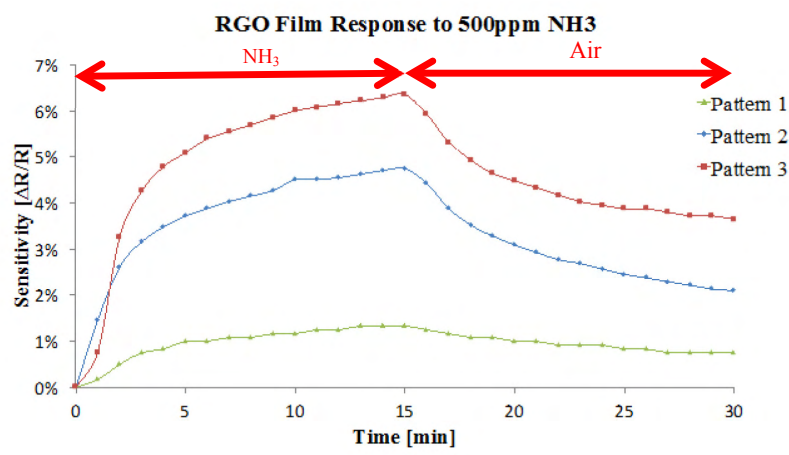

Figure 7. Measured response of RGO thin films in presence of $\mathrm{NH}_{3}$.

The increase in resistance upon the exposure to $\mathrm{NH}_{3}$ is due to the electron donation of $\mathrm{NH}_{3}$ to the p-type $\mathrm{RGO}$ film, and is consistent with the literature. Compared to other works on graphene-based gas sensors, our inkjet printed RGO demonstrates superior performance, including a high sensitivity, fast response and quick recovery. Particularly, the short recovery time in natural environmental conditions is hugely advantageous over other reports which used UV and heat treatment to assist the recovery, and is of great importance for practical applications [6]. The sensor exhibits a $10 \Omega$ change in resistance within one minute after introducing $\mathrm{NH}_{3}$, a measure which is certainly in the detectable range of the backend circuitry of the WISP platform. It is worthy to note here that although the absolute value of the resistance varies between samples, probably due to variances during fabrication, this has negligible influence on the overall performance of the sensor since only the relative changes in resistance are considered here, which are stable between samples.

\section{IMPROVEMENT OF SENSOR FABRICATION}

\section{A. Fabrication via Inkjet Printing on Si/glass}

The current efforts to improve sensor performance target improved thin film design. For this purpose, we are now actively depositing the thin films onto $\mathrm{Si} /$ glass substrate. Inkjet printing on $\mathrm{Si} /$ glass substrate is a challenge in inkjet printing technology. Due to the poor wettability of the GO ink on the smooth surface of $\mathrm{Si}$ wafer or glass, it has been difficult to print GO with fine patterns on such substrates. As $\mathrm{Si}$ (which has a thin film of $\mathrm{SiO}_{2}$ on its surface) and glass both have negatively charged surfaces in water, both $\mathrm{Si}$ and glass expel (negative) GO ink as a result. In order to facilitate the binding of GO to $\mathrm{Si}$ wafer and glass surfaces, surface modification to the substrates was performed prior to $\mathrm{GO}$ deposition. Briefly, hydroxyl groups on the $\mathrm{Si}$ or glass surfaces were used to introduce amine groups, which were strongly positively charged in water, to the substrate surfaces by a simple chemical reaction (Fig. 8a). The surface amine groups were then amplified via a layer-by-layer amplification process (Fig. 8b). The enriched surface positive charges were then ready to bind the negatively charged GO ink.

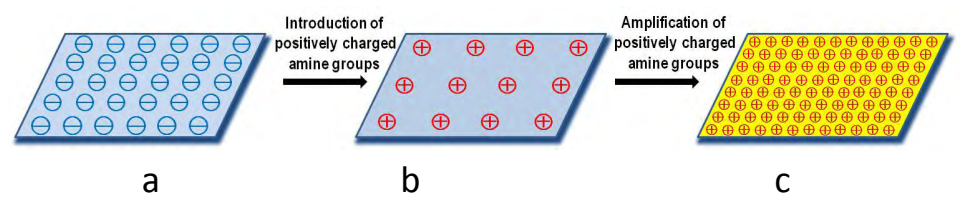

Figure 8. Surface modification of Si wafer or glass.

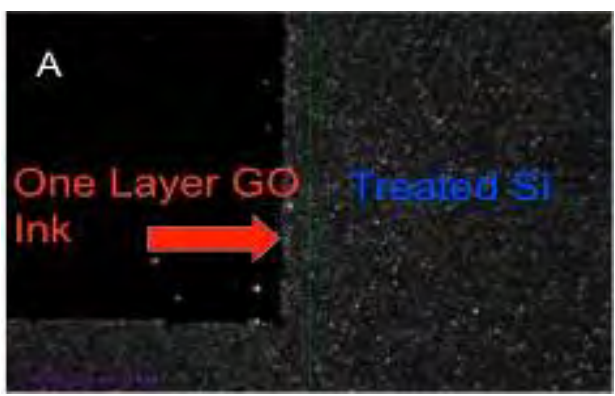

Figure 9. Measurement setup of the graphene sample.

This technology has significantly improved single/few layer print quality on smooth $\mathrm{Si} /$ glass (Fig. 9). It will allow many more investigative tools to verify the quality of RGO thin film. This work is expected to substantially increase thin film performance.

\section{B. Improved Reduction Methods}

The process of reduction is critical to achieve good quality of graphene. We are trying chemical, thermal and laser reduction methods to compare the printed RGO thin film by AFM, SEM and Raman spectra.

\section{ACKNOWLEDGMENT}

The authors would like to thank NSF-ECS, IFC-SRC and Intel WISP Challenge team for their support for the research.

\section{REFERENCES}

[1] G. Chen et al., "Enhanced gas sensing in pristine carbon nanotubes under continuous ultraviolet light illumination", Nature, Scientific Reports 2, Article number:343, March 2012.

[2] Z. Lin et al., "Solvent Assisted Thermal Reduction of Graphite Oxide," The Journal of Physical Chemistry C. pp14819-14825, 2010.

[3] Z. Lin et al., "Ultrafast dry microwave synthesis of graphene sheets." J.Mater. Chem., 2010, 20, pp.4781-4783.

[4] W. S. Hummers and R. E. Offeman, "Preparation of Graphitic Oxide," Journal of the American Chemical Society, vol. 80,(1958) pp. 13391339.

[5] A. Sample et al., "Design of an RFID-based battery-free programmable sensing platform," IEEE Transactions on Instrumentation and Measurement, pp. 2608-2615, 2008.

[6] F.Schedin et al., "Dection of individual gas molecules adsorbed on graphene." Nature Materials Vol.6, 2007. 